\title{
A Lurking Raging Infection in an Apparent Case of ANCA Vasculitis
}

\author{
Syed Rafay Ali Sabzwari¹, Mohammad Wazir², Syed Askari Hasan², Nimra Khan² and Aadil Maqsood ${ }^{3}$ \\ ${ }^{1}$ Lehigh Valley Health Network, USA \\ ${ }^{2}$ Florida Hospital, USA \\ ${ }^{3}$ Geisinger Medical Center, USA
}

Submission: December 26, 2017; Published: January 22, 2018

*Corresponding author: Syed Rafay Ali Sabzwari, Cardiology Department, Lehigh Valley Health Network 1250 S. Cedar Crest Blvd, Suite 300, Allentown, PA 18103, USA, Email: Rafay.Sabzwari@lvhn.org

\begin{abstract}
The association between Infective Endocarditis (IE) and positive testing for Anti-Neutrophil Cytoplasmic Antibody (ANCA) can raise diagnostic as well as treatment dilemmas. This is a case of a 39 year old Caucasian female without history of intravenous drug abuse who presented with worsening bilateral leg painful and purpuric rash for 1 week. Laboratory data was significant for normal CBC, white count and complement levels whereas creatinine was $4.49 \mathrm{mg} / \mathrm{dl}$ with a normal baseline, hepatitis C positive, C-ANCA titer 1:40 (anti-proteinase 3 positive) and urinalysis with $1+$ blood and protein. Patient was presumptively treated as ANCA vasculitis with high dose steroids as a kidney biopsy could not be obtained due to thin cortex on renal ultrasound.

Three days later she developed shortness of breath and chest x-ray revealed nodular opacities triggering CT chest that showed possible septic emboli together with a new 3/6 systolic murmur at left parasternal area. Blood cultures were positive for MRSA and transthoracic echocardiogram revealed moderate to severe tricuspid regurgitation with possible vegetation later confirmed on transesophageal echocardiogram. Steroids were immediately replaced by vancomycin resulting in complete recovery of rash and bacteremia by five weeks. Infective endocarditis is known to produce a wide variety of clinical manifestations. A few cases in which IE presented as ANCA vasculitis have been reported.
\end{abstract}

Keywords: Endocarditis; ANCA; Vasculitis; Glomerulonephritis; Hepatitis C; Cryoglobulinemia

\section{Introduction}

Infective endocarditis has been known to be associated with immune complex vasculitis which can manifest as cutaneous small vessel vasculitis, glomerulonephritis, Osler nodes and Roth spots. However, part of this immune complex mediated mechanism is more commonly positive rheumatoid factor and rarely C-ANCA. As C-ANCA itself can be associated with vasculitis affecting skin and kidneys, the underlying presence of infective endocarditis, a significant life-threatening infection, can be missed. Therefore the association between Infective Endocarditis (IE) and positive testing for Anti-Neutrophil Cytoplasmic Antibody (ANCA) can raise diagnostic as well as treatment dilemmas. This is a case of infective endocarditis closely mimicking ANCA-associated vasculitis and glomerulonephritis in a patient with HCV and negative cryoglobulins.

\section{Case Presentation}

A39year old Caucasian female with no significant pastmedical history presented with worsening bilateral lower extremity painful and purpuric rash for 1 week. She was having dark urine and back pain for two weeks that was treated as urinary tract infection with ciprofloxacin for 10 days without improvement.
On examination her vital signs and cardiopulmonary exam were unremarkable and palpable purpura on both legs was present. Laboratory data was significant for normal CBC, white count, cryoglobulin and complement levels whereas creatinine was $4.49 \mathrm{mg} / \mathrm{dl}$ with a normal baseline, hepatitis C was positive, C-ANCA titer 1:40 (anti-proteinase 3 positive) and urinalysis with $1+$ blood and protein. Of note renal histology was not attempted as a renal ultrasound obtained upon admission demonstrated cortical thinning in her kidneys that raised the risks associated with biopsy considerably and a low diagnostic yield. However, the patient's presentation along with early lab findings were highly suggestive of ANCA associated vasculitis and cryoglobulinemia, likely related to HCV. High dose steroids in the form of $1 \mathrm{~g}$ methylprednisone for three days were given empirically.

Patient improved significantly for next three days but later developed slight shortness of breath and chest x-ray revealed nodular opacities triggering CT scan of the chest that showed septic emboli over both lung fields (Figure 1). In addition, physical exam then was significant for a faint $2 / 6$ systolic murmur at left sternal border, and an EKG showed 
left anterior hemiblock. Three sets of blood cultures were sent and all came back positive for methicillin resistant Staphylococcus aureus (MRSA) which warranted a transthoracic echocardiogram that revealed moderate to severe tricuspid regurgitation with possible vegetation of $5 \times 3 \mathrm{~mm}$ (Figure 2). This was confirmed later on transesophageal echocardiogram. At the same time, the cryoglobulin level came back as negative excluding cryoglobulinemia as a possibility. Indeed, the patient's presentation satisfied the major Duke criteria for Infective Endocarditis.

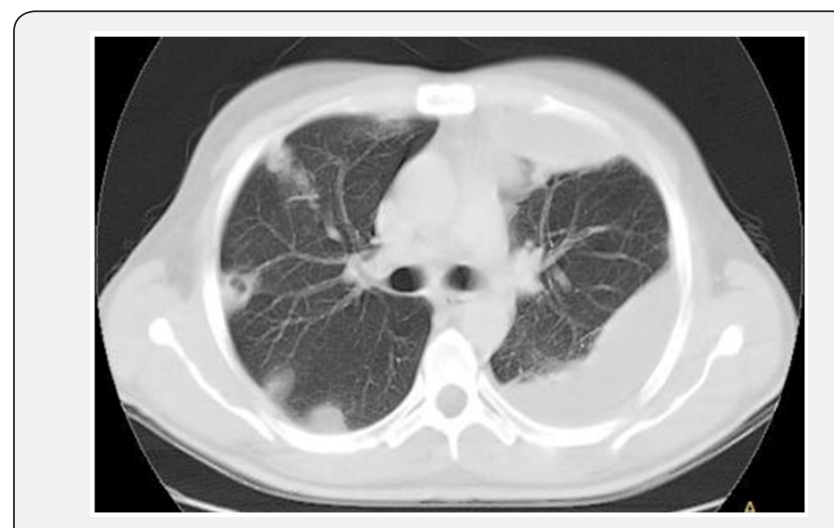

Figure 1: CT scan of chest with bilateral nodular lung opacities representing septic emboli.

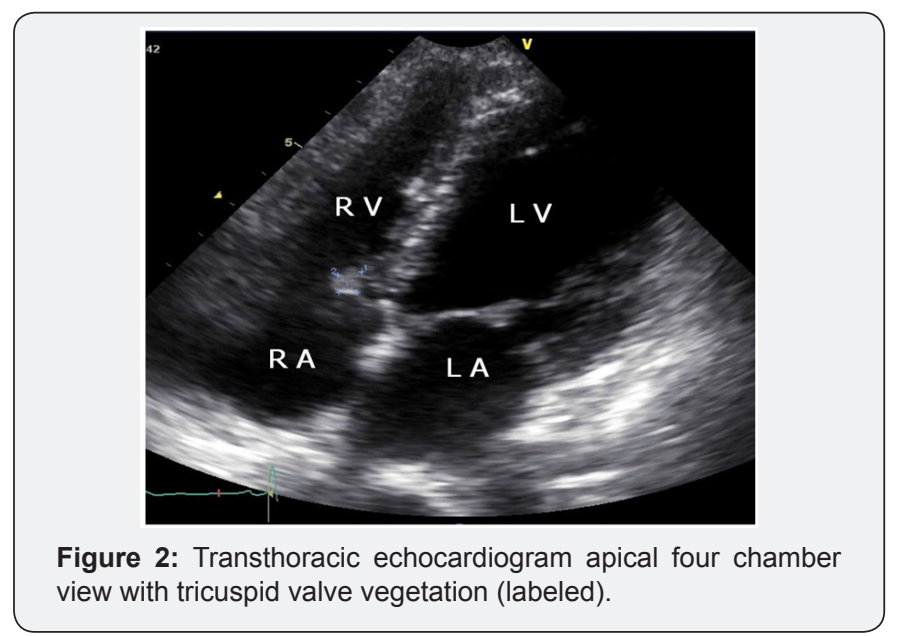

Steroids were stopped immediately after getting three days of treatment and were started on vancomycin. Repeat blood cultures after forty-eight hours with vancomycin were negative and patient's rash and symptoms started to improve as well.
Therefore, to ease administration of outpatient antibiotics, he was changed to daptomycin which was continued at home for further four weeks. On follow up at five weeks the skin rash had almost completely resolved and did not have significant symptoms. A TTE was repeated which showed stable moderate to severe tricuspid regurgitation. As the right ventricular size and function was intact with no significant volume overload signs it was decided to monitor the tricuspid regurgitation periodically.

\section{Discussion}

A number of cases in which infective endocarditis presented identically to autoimmune syndromes have been reported in the literature $[1,2]$. In the case presented, initially an audible murmur on cardiac auscultation, other signs suggestive of infection and intravenous drug use behavior were absent which lessened the suspicion of an underlying infection. The other confounders were the positive HCV and C-ANCA result that could be attributable to many of patient's clinical manifestations. Other cases in literature, along with the case presented in this report, were initially misdiagnosed as a result, and managed with corticosteroids and other immunosuppressive regimens, which unfortunately prompted further deterioration of the patient's condition before conclusive evidence of infective endocarditis could be found.

This phenomenon is not confined to infective endocarditis but to other systemic infections mainly those with significant bacteremia that leads to immune complex formation triggering downstream immunologic cascades. The exact pathophysiology behind this is unclear at this point [1]. In one study, the prevalence of c-ANCA and/or p-ANCA in patients with IE was found to be $18 \%$ [2]. However, it does imply that a greater clinician awareness of the circumstances and timing surrounding proper exclusion of bacterial endocarditis from a patient's differential would likely be of benefit. Also that in cases where a clear focus of infection cannot be found, at least a set of cultures should be drawn as most of these states have profound bacteremia.

\section{References}

1. Peng H, Chen WF, Wu C, Chen YR, Peng B, et al. (2012) Culture-negative subacute bacterial endocarditis masquerades as granulomatosis with polyangiitis (Wegener's granulomatosis) involving both the kidney and lung. BMC Nephrol 13: 174

2. Riding AM, D'Cruz DP (2010) A case of mistaken identity: subacute bacterial endocarditis associated with p-antineutrophil cytoplasmic antibody. BMJ Case Rep pii: bcr0920103299. 
This work is licensed under Creative

Commons Attribution 4.0 License

DOI: 10.19080/OROAJ.2018.10.555782

\section{Your next submission with Juniper Publishers} will reach you the below assets

- Quality Editorial service

- Swift Peer Review

- Reprints availability

- E-prints Service

- Manuscript Podcast for convenient understanding

- Global attainment for your research

- Manuscript accessibility in different formats ( Pdf, E-pub, Full Text, Audio)

- Unceasing customer service

Track the below URL for one-step submission https://juniperpublishers.com/online-submission.php 